\title{
Study on the Electromagnetic Driving Modal and Control of Spherical Driving Joint with Magnetic Levitation
}

\author{
Zhang Fan ${ }^{\text {a, }}$, Zeng Li $^{\text {a }}$, Zhu Zhida ${ }^{\mathrm{a}}$ \\ ${ }^{a}$ College of Mechanical Engineering Yangzhou University, Yangzhou 225127, China \\ *Corresponding Author: fzhang@yzu.edu.cn
}

\begin{abstract}
The multi-degree-of-freedom spherical driving joint with direct bearing driven by spherical motor owns high mechanical integrity and has many advantages such as control and trajectory planning. This paper presents a novel multi-degree-of-freedom spherical reluctance driving joint with magnetic levitation, and analyses operation mechanism, and next deduces the joint's magnetic conduction of the gap by means of the way to magnetic segmentation achieves the relationship between electrical and mechanical energy conversion, and then calculates the radial suspension force and the tangential suspension torque which spherical driving joint produces. The simulation experiments show that the system of driving joint has well static and dynamic characteristics.
\end{abstract}

Keywords: Spherical Driving Joint, Magnetic Suspending Force, Electromagnetic Torque, Control Characters.

\section{Introduction}

The movement oh multi-freedom joints is usually composed of several single-degree-of-freedom joints through coordination, translation and rotation of the connecting rod, It often needs more single-degree-offreedom driving mechanism and complex mechanical transmission mechanism to finish ${ }^{[1]}$. Therefore, a multi-freedom joint system exists not only the complex structure, the huge size, the excessive wear of the joint friction surface, the low efficiency, the difficult manufacture and installation, the slow response and the bad dynamic performance, etc.; but also due to the accumulation of mechanical transmission system error, the precision of control system fell and it even influences the stability of system ${ }^{[2]}$. From the beginning of the 1950 s, due to the rapid development of the robot, space technology and automation technology, it promotes the development of the motor principle of the multi-degree-of-freedom spherical rotor and the research of the structure ${ }^{[3-5]}$. The driving joint system which based on the spherical driving joint with direct bearing driven by spherical motor is the multi-degree-of-freedom spherical driving joint, it owns high mechanical integrity and instead of two or more single-degree-of-freedom motor , a more freedom spherical joints can drives transmission mechanism which is made up of multi-freedom joints, it simplify the structure of the mechanical system and reduce the volume and transmission chain, weight, eliminate the gear tooth gap and the friction, etc, so as to improve the efficiency of system, the accuracy ,the dynamic performance and high cost performance, have the advantage in the trajectory planning and in control. But in order to realize the spherical motor rotating at different degrees, it generally uses the frame structure, each layer frame is supporting by mechanical bearing to spin around a coordinate, so it exist complex structure, large size and the restricted rotation angle, etc. When in the high speed and even ultra-high speed of the multi-degree-of-freedom joint device, due to friction of mechanical (bearing) bearing, mechanical friction not only increased the joint friction, but also which generates heat, it even makes dynamic characteristics of the driving joint bad. Based on motor technology, magnetic levitation and robotics, the paper presents a novel multi-degree-offreedom magnetic levitation spherical reluctance driving joints which domestic haven't report, it uses magnetic levitation force to make spherical rotor suspend, makes it no friction with the stator and no abrasion, has high 
accuracy and good dynamic performance ${ }^{[6-7]}$; and make mechanism study for it to produce the magnetic levitation force and the magnetic torque.

\section{2. the Basic Components of Magnetic Levitation Spherical Driving Joint}

Under the active control of the controller, the magnetic levitation spherical driving joint can produce the electromagnetic levitation force which is supporting for the joint rotor and the electromagnetic torque which drive the joint rotor rotating, it owns two or three rotating freedom and takes rotating work with non-contact and abrasion free bypass the fixed point of the space axes. It is mainly made up of several motor stator driving joint and the spherical driving joint rotor with the tumbler, etc., and the three-dimensional structure is shown in Fig. 1. The joint rotor is surrounded by the joints of the motor stator, the four stators are symmetrical distribution at the equatorial line position, the stator 1 and 3 are symmetry for spherical rotor and keep coaxial along the $\mathrm{X}$-axis, its effect is to drive the rotor rotating around the $\mathrm{X}$-axis and make rotor produce the stability magnetic levitation force in the $\mathrm{X}$-axis; the stator 2 and 4 are symmetry for spherical rotor and keep coaxial along the Y-axis, its effect is to drive the rotor rotating around the $\mathrm{Y}$-axis and make rotor produce the stability magnetic levitation force in the Y-axis; the stator 5 arranges the top of spherical rotor and keeps coaxial with the Z-axis, its function is driven rotor spinning around the $\mathrm{Z}$-axis and make the rotor produce the stability magnetic levitation force in the Z-axis.

Some shapes of saliency column distributed in the outside surface of magnetic levitation spherical joint (cylinder or Prism Object), the capital envelope spherical surface is rotor spherical surface; The stator joint contains a number of similar saliency shape salient pole, and
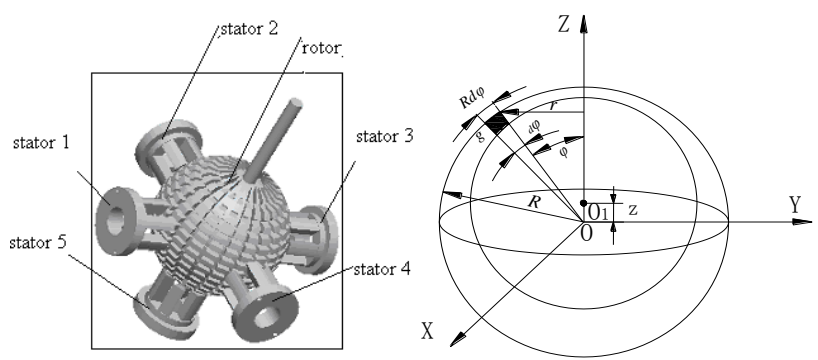

Fig.1 Structure drawing of spherical driving joint

Fig.2 Schematic diagram of with maonetic levitation according to principle of reluctance motor, multi-phase (m) winding winds around the salient pole which drives the rotor rotating, each phase windings have a pole. Each phase windings after electrify produce electromagnetic torque to drive the spherical rotor and also provides the radial levitation force for the spherical rotor.

\section{The air-gap magnetic conductance and energy of Magnetic Levitation Spherical reluctance Driving Joint}

Based on the spherical reluctance motor drive and support the spherical rotor with the tumbler, the magnetic levitation spherical reluctance driving joint realizes multi-freedom joints. Therefore, the study is the same of other analysis method of the reluctance, from the air-gap of the motor stator and rotor, the mechanical energy transformational relation is established, and then it get the relation between the electromagnetic levitation force which drives rotor suspending and electromagnetic torque which drives the rotor rotating.

If the center of sphere of the magnetic spherical reluctance driving joint doesn't happen excursion, the radius of the stator salient pole envelope spherical surface is $R$, the radius of the rotor salient pole envelope spherical surface is $R_{r}$, the clearance between the stator salient pole envelope spherical surface and the rotor salient pole envelope spherical surface is $g_{0}=R-R_{r}$. Make the symmetry axis of the stator to coincide with $\mathrm{Z}$ axis and build frame of axes $f(X, Y, Z)=F(R, \varphi, \theta)$, as shown in Fig. 2. When the rotor joint is in the process of rotating, its salient pole surface is the overlap alignment with stator salient pole surface and it produces magnetic-pull (thrust) force to form electromagnetic torque, magnetic-pull force is the magnetic levitation force which drives the rotor suspend. Magnetic-pull (thrust) force is directly related to the size and distribution of the air-gap magnetic conductance.

If the joint rotor is under the action of the interference, the centre-of-sphere of the rotor shifts from $O$ to $O_{1}$, the offset is $z$ along the Z-axis, when the angle is $\theta$ which rotated around the $\mathrm{Z}$-axis, the gear upper surface of the stator salient pole and the rotor salient pole will be stagger in the direction of the rotor offset (front, back) and the rotor rotating(left, right),it likes the Fig. 3. By using the method of the air-gap magnetic field segmentation to calculate magnetic conductance, it is divided into five parts, there are the magnetic conduction P0 of the overlap between the stator and the magnetic top of the rotor as shown in the Fig. 


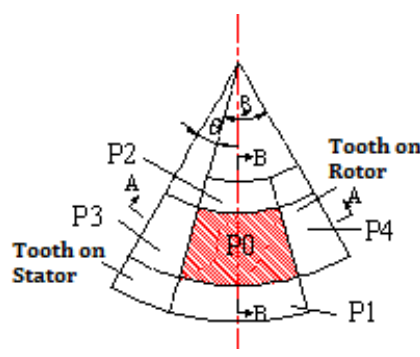

(a) the distribution of the magnetic conductance

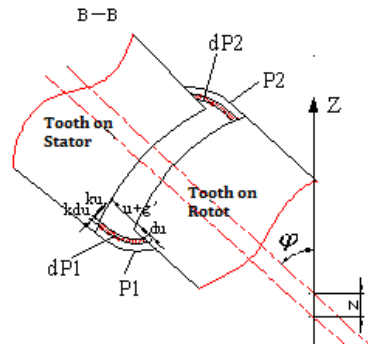

(c) the rotor offset to cause the edge magnetic permeance

Fig.3 Magnetic paths partition of air gap

3(a),the edge magnetic (left flank and right flank) conduction P3. P4 which is a cause for the rotating rotor as shown in the Fig. 3(b); the edge magnetic (front flank and back flank) conduction P1. P2 which is a cause for the offset of the rotor as shown in the Fig. 3(c). The total magnetic conduction of this mutual interaction between the air-gap magnetic is:

$$
P(z, \theta)=P_{0}+P_{1}+P_{2}+P_{3}+P_{4}
$$

Because the departure of the rotor from the equilibrium position is very small, the surface between the stator and the magnetic the rotor salient pole is considered the parallel surface, the magnetic circuit of the overlap between the stator gear and the rotor gear can be instead of the linear magnetic circuit; the elliptic curve approximately replaces the edge magnetic flow path $^{[8-9]}$.after simplifying like this, the calculation of the total magnetic conduction of the interaction between the pair poles for air-gap is:

$$
\begin{aligned}
& P(z, \theta)=\frac{\mu_{0}(L-r \theta)(L-z \sin \varphi)}{g}+\frac{2 \mu_{0}}{\pi}(L-r \theta) \ln \frac{2 z^{2} \sin ^{2} \varphi+2 g z \sin \varphi+g^{2}}{g^{2}} \\
& +\frac{2 \mu_{0}}{\pi}(L-z \sin \varphi) \ln \frac{g^{2}+2 g r \theta+2 r^{2} \theta^{2}}{g^{2}}
\end{aligned}
$$

Where $\mu_{0}$ is the space permeability, $r$ is the inside circular arc radius of the stator magnetic pole, $L$ is the side length of the stator tooth notching, $\varphi$ indicates the angle between the position of the stator tooth shape at normal and Z-axis, $g=g_{0} \mathrm{~m} z \cos \varphi$ is the radial air gap length between the crest top land of the rotor salient pole and the crest top land of the stator salient pole(magnetic pole), when the rotor near the stator, to take "- ", when the rotor depart from the stator, to take" $+", g_{0}$ is the air-gap radius between the magnetic pole and the stator magnetic pole when the rotor doesn't have offset.

If the stator motor joint has $m$ phase windings, each phase winding has a magnetic pole to work with electricity, according to the theory of motor, the calculation of the total energy which is stored in the air-gap magnetic field of the joint stator motor is:

$$
W_{g}=\frac{1}{2} L m i^{2}=\frac{1}{2} K m N^{2} i^{2} P(z, \theta)
$$

Where $N$ is turns per coil of one phase winding, $L=K N^{2} P(z, \theta)$ is the inductance which one phase winding generates.

When the spherical rotor makes the offset along $\mathrm{X}$-axis or Y-axis, similarly it can deduce the magnetic conduction of the joint air gap and the total magnetic energy which is the same structure of the equation 2 and the equation 3 . According to the magnetic energy $W_{g}$ of the joint air gap, the magnetic levitation force which the joint generates can be taken by making the derivation of the offset of the rotor along the coordinate, the electromagnetic torque of the driving rotor which the joint generates can be taken by making the derivation of the rotor angle $\theta$.

\section{4. the Magnetic levitation force and the electromagnetic torque of the Magnetic Levitation Spherical Reluctance Driving Joint}

According to equation 3, it can take the magnetic levitation force and the electromagnetic torque of the magnetic levitation spherical reluctance driving joint along the Z-axis by making the partial derivative of $z$ and $\theta$ :

$$
\left\{\begin{array}{l}
F_{z}=\frac{1}{2} K m N^{2} i^{2}\left[\frac{\mu_{0}(L-r \theta)[g(-\sin \varphi)-(L-z \sin \varphi)(\mu \cos \varphi)]}{g^{2}}+\right. \\
\frac{4 \mu_{0}(L-r \theta)(2 z \sin \varphi+g) g_{0} \sin \varphi}{\pi g\left(2 z^{2} \sin ^{2} \varphi+2 g z \sin \varphi+g^{2}\right)}-\frac{2 \mu_{0} \sin \varphi}{\pi} \ln \frac{g^{2}+2 g r \theta+2 r^{2} \theta^{2}}{g^{2}} \\
\left.+\frac{\left.4 \mu_{0} r \theta(L-z \sin \varphi)(g+2 r \theta)(\mu \cos \varphi)\right]}{\pi g^{3}}\right] \\
T_{z}=\frac{1}{2} K m N^{2} i^{2}\left[\frac{4 \mu_{0} r(L-z \sin \varphi)(g+2 r \theta)}{\pi\left(g^{2}+2 g r \theta+2 r^{2} \theta^{2}\right)}-\frac{\mu_{0} r(L-z \sin \varphi)}{g}-\right. \\
\left.\frac{2 \mu_{0} r}{\pi} \ln \frac{2 z^{2} \sin ^{2} \varphi+2 g z \sin \varphi+g^{2}}{g^{2}}\right]
\end{array}\right.
$$

According to the equation 4, through controlling the supply current of the joints, it can adjust the size of magnetic levitation force and the electromagnetic torque.

For the $\mathrm{X}$-axis (Y-axis) direction, due to the two stator electromagnet driving joint rotor to move, so its magnetic 
levitation force is:

$F_{x}=F_{x 1}-F_{x 2}$

$=\frac{1}{2} K m N^{2} i_{2}^{2}\left[\frac{\mu_{0}(L-r \theta)\left[g_{x 1}(-\sin \varphi)-(L-x \sin \varphi) \cos \varphi\right]}{g_{x 1}^{2}}+\right.$

$\frac{4 \mu_{0}(L-r \theta)\left(2 x \sin \varphi+g_{x 1}\right) g_{0} \sin \varphi}{\pi g_{x 1}\left(2 x^{2} \sin ^{2} \varphi+2 g_{x 1} x \sin \varphi+g_{x 1}^{2}\right)}-\frac{2 \mu_{0} \sin \varphi}{\pi} \ln \frac{g_{x 1}^{2}+2 g_{x 1} r \theta+2 r^{2} \theta^{2}}{g_{x 1}^{2}}$

$\left.+\frac{\left.4 \mu_{0} r \theta(L-x \sin \varphi)\left(g_{x 1}+2 r \theta\right) \cos \varphi\right]}{\pi g_{x 1}^{3}}\right]-$

$\frac{1}{2} K m N^{2} i_{2}^{2}\left[\frac{\mu_{0}(L-r \theta)\left[g_{x 2}(-\sin \varphi)-(L-x \sin \varphi) \cos \varphi\right]}{g_{x 2}^{2}}+\right.$

$\frac{4 \mu_{0}(L-r \theta)\left(2 x \sin \varphi+g_{x 2}\right) g_{0} \sin \varphi}{\pi g_{x 2}\left(2 x^{2} \sin ^{2} \varphi+2 g_{x 2} x \sin \varphi+g_{x 2}^{2}\right)}-\frac{2 \mu_{0} \sin \varphi}{\pi} \ln \frac{g_{x 2}^{2}+2 g_{x 2} r \theta+2 r^{2} \theta^{2}}{g_{x 2}^{2}}$ $\left.+\frac{\left.4 \mu_{0} r \theta(L-x \sin \varphi)\left(g_{x 2}+2 r \theta\right) \cos \varphi\right]}{\pi g_{x 2}^{3}}\right]$

The air gap between the two stator and rotor magnetic poles is: $g_{x 1}=g_{0}-x \cos \varphi, g_{x 2}=g_{0}+x \cos \varphi$. Similarly the total electromagnetic torque of the two joints stator which drives the spherical rotor rotating is:

$$
\begin{aligned}
& T_{x}=T_{x 1}+T_{x 2} \\
& =\frac{1}{2} K m N^{2} i^{2}\left[\frac{4 \mu_{0} r(L-x \sin \varphi)\left(g_{x 1}+2 r \theta\right)}{\pi\left(g_{x 1}^{2}+2 g_{x 1} r \theta+2 r^{2} \theta^{2}\right)}-\frac{\mu_{0} r(L-x \sin \varphi)}{g_{x 1}}-\right. \\
& \frac{2 \mu_{0} r}{\pi} \ln \frac{\left.2 x^{2} \sin ^{2} \varphi+2 g_{x 1} z \sin \varphi+g_{x 1}^{2}\right]+\frac{1}{2} K m N^{2} i^{2}\left[-\frac{\mu_{0} r(L-x \sin \varphi)}{g_{x 1}^{2}}-\right.}{\left.\frac{2 \mu_{0} r}{\pi} \ln \frac{2 x^{2} \sin ^{2} \varphi+2 g_{x 1} z \sin \varphi+g_{x 1}^{2}}{g_{x 1}^{2}}+\frac{4 \mu_{0} r(L-x \sin \varphi)\left(g_{x 1}+2 r \theta\right)}{\pi\left(g_{x 1}^{2}+2 g_{x 1} r \theta+2 r^{2} \theta^{2}\right)}\right]}
\end{aligned}
$$

According the equation 5 and 6, the magnetic levitation force and electromagnetic torque mutually interference and influence which the joint generates, the whole system is the nonlinear strong coupled system and needs nonlinear decoupling control.

\section{5. the control system and the simulation analysis of the Magnetic Levitation Spherical reluctance Driving Joint}

\section{1 the principle of the control system}

In the same coordinate direction, two reluctance motor stator windings of magnetic levitation spherical reluctance driving joints can be alternative controlled according to the control strategy of the reluctance motor, the subsystem which constitutes two reluctance motor can synchronous drive sphere rotor rotating. Take two of the three-phase $(\mathrm{m}=3)$ motor driving in the $\mathrm{X}$-axis for example, It detects the offset signal of the rotor by displacement sensor, to convert it into current (or voltage signals) by after filtering and amplification and suspension controller, etc,

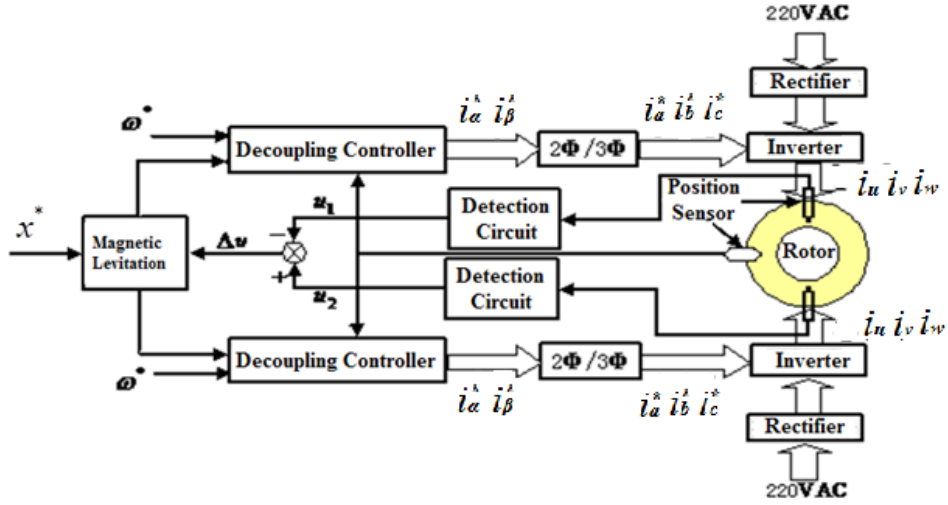

Fig.4 Control principle of spherical driving joint with magnetic levitation

after making feedback to the input of the windings control and comparing it with the displacement of the input signals $x^{*}$, then to go through decoupling controller decoupling with the rotate speed signals of the rotor and transform the decoupling signal into the control signals of the three-phase circuit by $2 \Phi / 3 \Phi$, so as to change the current size of the stator winding and achieve suspension control and rotating control, the principle of control system is shown in Fig. 4.

\section{2 the analysis of control simulation results}

According to the voltage equation of the joint stator winding and the kinematics equations of the spherical rotor, the nonlinear coupling dynamic mathematical model of magnetic levitation spherical reluctance driving joint, as shown in the Fig. 4, the control system begins to emulate magnetic levitation spherical reluctance driving joint and then can analyze the performance of the system.

(1) the simulation results of the joint rotor which is disturbed

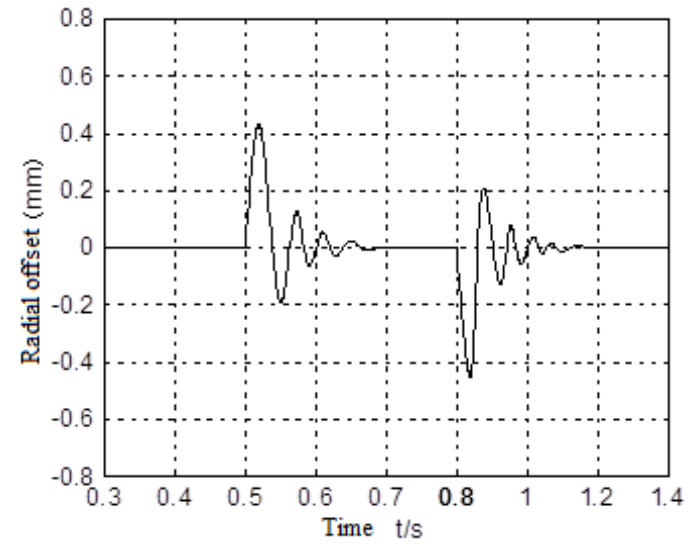

Fig.5 Radial offset with interference of Laplace domain signals

After the driving joints achieved stabilize suspension, the simulation result of impulse interference in the positive and negative direction is shown in Fig. 5 which the joint 
rotor input when it is at 0.5 seconds and 0.8 seconds. According to the figure, if the magnetic levitation spherical reluctance driving joint received the radial disturbance, it can restore to the equilibrium position in a short period of time, the rising time is all under 0.2 seconds and the overshoot is small; it doesn't have the shocking phenomenon.

(2) the rotating speed response of the spherical rotor

After the inverse system decoupling and comprehensive correction, Fig. 6 shows the rotating speed (angular velocity) step response of the spherical rotor spinning around the $\mathrm{X}$-axis, the expected rotating speed is $1.2 \times 10^{5}$ $\mathrm{r} / \mathrm{min}$. According to the results of the simulation and the rotating has less overshoot, the settling time is $0.5 \mathrm{~s}$, the subsystem of the rotating speed has good performance index

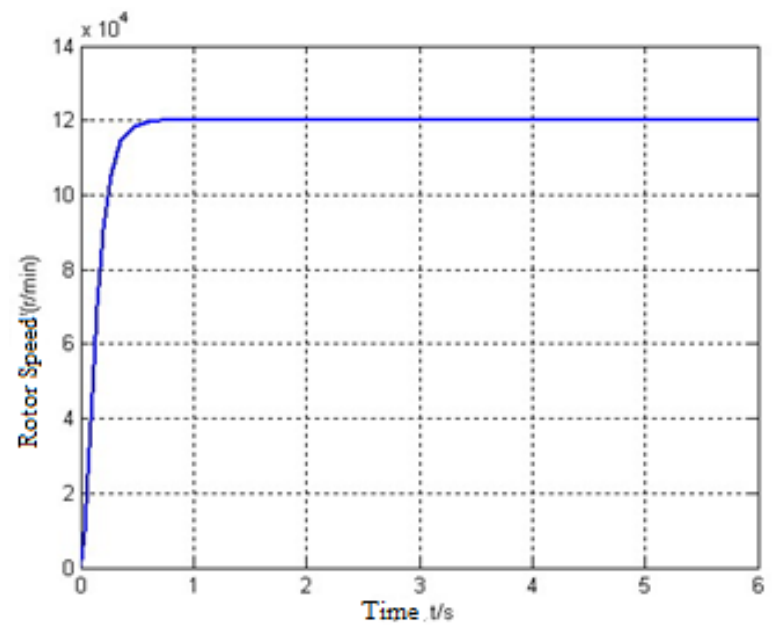

Fig.6 The dynamic response of the rotor speed

\section{Conclusions}

This paper presents the multi-degree-of-freedom magnetic levitation spherical reluctance driving joint which owns the simple structure, high precision and good dynamic performance; According to the air-gap magnetic energy, it established the transformational relation of the joint mechanical energy and studies the mechanism of the joint to produce electric magnetic levitation force and electromagnetic torque; according to this, the comprehensive control system of the joint is established and it made the system simulation analysis of it, the simulation results show that the driving joint system has good dynamic and static performance and the anti-interference ability.
Thank the China Natural Science Foundation for this project. Item Number: 51375427. Thank the Jiang Su Natural Science Foundation for this project. Item Number: BK20131232. Thank the Jiang $\mathrm{Su}$ Natural Science Foundation for this project. Item Number: BY2014117-08, BY2015061-04.

\section{References}

(1) Wang Guangjian, Liang Xichang, Jiang Jiandong: The Present State and Developing Tendency of Robot Joint. Journal of Mechanical Transmission, 2004.28(4):1-5.

(2) Wu Junfei, Zhou Guilian, Fu Ping: Research Progress of Drives Used in Robot Joint. Journal of Qingdao Institute of Chemical Technology, 2002.23(3):54-58.

(3) Davey K, Vachtsevanos G, Powers R: The analysis of fields and torques in spherical induction motors. IEEE Trans. on Magn, 1987, 23(1):273-282.

(4) Kaneko K, Yamada I, Itao K.: A spherical DC servo motor with three degrees of freedom. ASME Trans. Dynamic Systems Measurement and Control, 1988, 111(3):398-402.

(5) Gregory S. Chirikjian, David Stein: Kinematic Design and Commutation of a Spherical Stepper Motor D Stein, G S Chirikjian. Experiments in the Commutation and Motion Planning of a Spherical Stepper Motor. ASME Int. Conf. DETC. September, 2000. 1-7.

(6) Zeng Li, Zhang Dan, Dai Min: Maglev Spherical Reluctance Motor with Centripetal Thrust/Pull. China, ZL200920039032.7, 2010.

(7) Zeng Li, Zhang Dan, Jiang Mingming: Switch Reluctance Driving Joint with Magnetic Levitation. China, ZL200920039031.2, 2010.

(8) M. Takemoto, A. Chiba, T. Fukao: A Feed-forward Compensator for Vibration Reduction Considering Magnetic Attraction Force in Bearingless Switched Reluctance Motors. Seventh International Sympsium on Magnetic Bearings. ETH Zurich,2000

\section{Acknowledgment}

